A. K. Dupree and A. O. Benz, Eds.

\title{
FIP Effect and FIP-Dependent Bias in the Solar Corona
}

\author{
B.N. Dwivedi, A. Mohan \\ Department of Applied Physics, Institute of Technology, Banaras Hindu \\ University, Varanasi-221 005, India \\ E. Landi \\ Artep, Inc. at Naval Research Laboratory, Washington, DC 20375, USA
}

\begin{abstract}
Using EUV spectra of an active region observed off the solar disk by the SOHO/SUMER spectrograph on the SOHO spacecraft, we investigate the dependence of the FIP effect on the height above the photosphere, and its relation to plasma magnetic structures present in the field of view. We also investigate the possibility of the FIP bias in the low-FIP elements to be FIP-dependent, so that different abundance anomalies must be found even within the low-FIP class of elements, which can provide important constraints on the FIP effect models.
\end{abstract}

\section{Introduction}

Elemental abundances are among the most fundamental sets of parameters in the solar and stellar atmospheres. Extensive research on the abundances of elements in the solar atmosphere has been updated in an excellent review by Feldman and Widing (2003). Abundances are often suggested to be correlated with the first ionization potential (FIP). Many studies show that a 'FIP bias' does exist, and an increase by a factor of four has been measured for elements with FIP lower than $10 \mathrm{eV}$ relative to those with FIP larger than $10 \mathrm{eV}$. Relative abundances within each FIP class have been assumed to be constant. So far, no theoretical model has been able to satisfactorily explain this effect.

In the present paper, we investigate the $\mathrm{Mg} / \mathrm{Ne}, \mathrm{S} / \mathrm{Ar}, \mathrm{Si} / \mathrm{Ar}$ and $\mathrm{K} / \mathrm{Ar}$ abundance ratios as a function of position in the off-disk solar corona. The field of view is composed of cold $\left(6 \times 10^{5} \mathrm{~K}\right)$ plasma structures embedded in a hotter unstructured plasma at $1.8 \times 10^{6} \mathrm{~K}$. Since the FIP of K, Si, Mg, S, Ar and $\mathrm{Ne}$ range from very low to high, the present study allows us to determine 1) the high-FIP/low-FIP ratio in the structured plasma using $\mathrm{Ne}$ VI and $\mathrm{Mg} \mathrm{VI} ; 2$ ) the $\mathrm{K} / \mathrm{Ar}, \mathrm{Si} / \mathrm{Ar}$ and $\mathrm{S} / \mathrm{Ar}$ relative abundance dependence with height; and 3) a FIP-dependent FIP bias for the low-FIP elements.

\section{Observations}

The observations were made with the SUMER spectrograph on 1996 June 20 above the active region NOAA 7974 at the solar east limb, in two $40 \AA$ wide 
Table 1. Ions and lines considered in the present work. $\log \mathrm{T}_{m}$ is the temperature of maximum abundance for each ion (in $\mathrm{K}$ ).

Ion Wvl. $(\AA) \quad \log \mathrm{T}_{m x} \quad$ FIP $(\mathrm{eV})$

\begin{tabular}{llrr}
\hline & & & \\
Mg VI & $1190.10,1191.64$ & 5.6 & 7.6 \\
Mg VII & 1189.85 & 5.7 & 7.6 \\
Ne VI & $992.73,997.14,999.26,1005.70,1010.25$ & 5.6 & 21.6 \\
& & & \\
K XIII & 994.58 & 6.4 & 4.3 \\
Si XI & 604.17 & 6.2 & 8.2 \\
S X & $1196.25,1213.00$ & 6.1 & 10.4 \\
Ar XII & 1018.87 & 6.4 & 15.8 \\
& & & \\
\hline
\end{tabular}

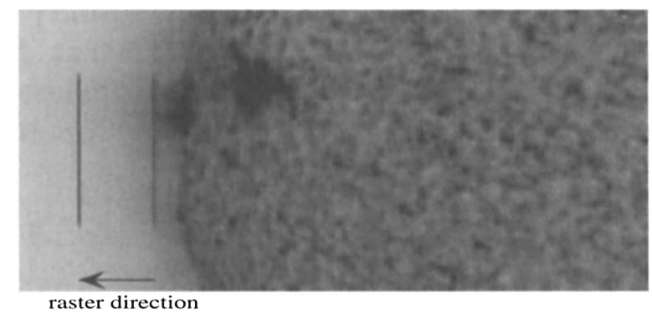

Figure 1. The SUMER field of view superimposed to the EIT $304 \AA$ channel, observing chromospheric plasma.

wavelength ranges centered on the Ne VI $999 \AA$ and Mg VI $1191 \AA$ line. A full description of SUMER is given in Wilhelm et al. (1995). In the observations analyzed in the present work a slit size of $4^{\prime \prime} \times 300^{\prime \prime}$ was used to raster a field of view from $40^{\prime \prime}$ to $173^{\prime \prime}$ above the east solar limb. The spatial resolution is $1^{\prime \prime}$ along the slit length. The integration time per spectrum was increased exponentially with position from $250 \mathrm{~s}$ at 40 " to $867 \mathrm{~s}$ at 173 ". The observed ions are listed in Table 1.

The observations analyzed in the present work include both plasma from active region structures and outside them. The field of view is shown in Figure 1, as seen by EIT, and Figure 2, as seen with S X and Mg VI. Mg VI shows a highly non-uniform behaviour, since this ion is confined inside a cool structured area belonging to the active region at the limb. Outside this area, S X presents a fairly uniform map with no signature of the active region. We refer to the low temperature plasma inside the structures as "prominence" plasma, and to the hotter coronal plasma as "active corona". Prominence plasma emits Ne VI and Mg VI lines, while the hotter plasma emits the other ions. In the present work these two plasmas have been analysed separately. 

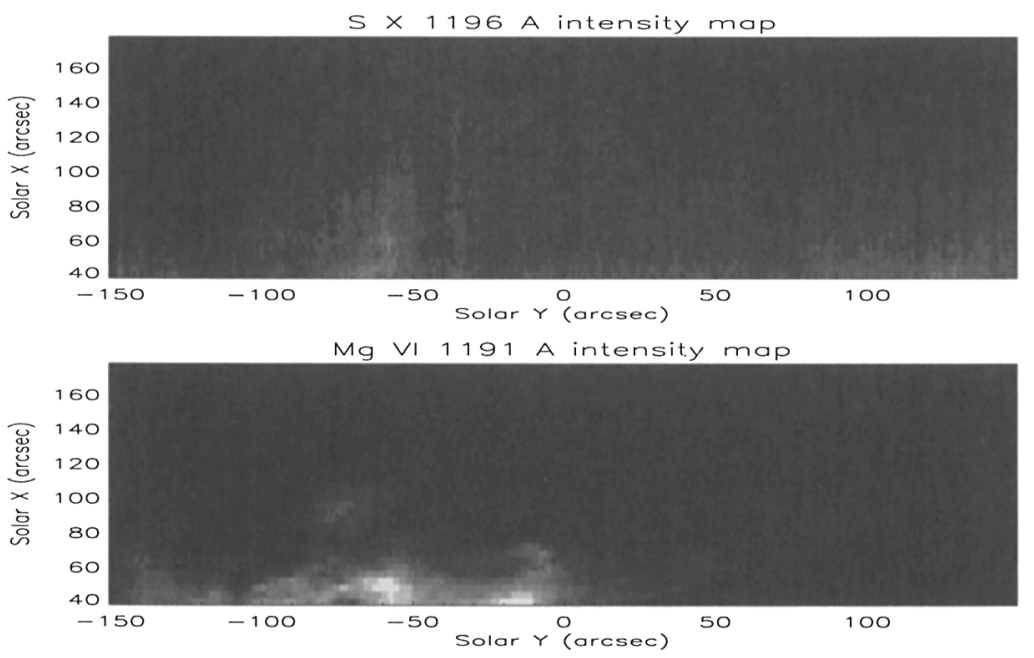

Figure 2. The SUMER field of view. Top: S X $1196 \AA$ (active corona); Bottom: Mg VI $1191 \AA$ (cool structures).

\section{Results}

Electron density and temperature were measured in both plasmas using line intensity ratios computed with the CHIANTI database (Dere et al. 1997) and their observed values.

Prominence plasma. The $1190 / 1191 \mathrm{Mg}$ VI line ratio shows that the $\log \mathrm{N}_{e}$ varies from 9.0 to $9.7\left(\mathrm{~N}_{e}\right.$ in $\left.\mathrm{cm}^{-3}\right)$ inside the bright structures. Each structure seems to have its own density. Outside the structures, the ratio increases and shows a greater noise, due to line weakness and instrument-scattered light. The Mg VII/Mg VI ratio, calculated at the measured density, shows that the prominence plasma is cooler and denser compared to the surrounding plasma. The variation of the $\mathrm{Mg} / \mathrm{Ne}$ relative abundance is correlated with the plasma structures. Some structures with strong Ne VI and Mg VI line emission indicate a normal $\mathrm{Mg} / \mathrm{Ne}$ FIP-bias between 1.6 to 3.2 ; In some other structures the $\mathrm{Mg} / \mathrm{Ne}$ FIP-bias ranges from 3.1 to 8.8 . These values show that the plasma structuring can have a strong effect on element abundances. An example is given in Figure 3, where the Ne VI intensity and the FIP bias is shown along the slit at solar $\mathrm{X}=45$.

Active coronal plasma. The 1196/1213 S X line ratio was used to determine $\mathrm{N}_{e}$ outside the cool structures. The electron temperature outside the cool structures was determined from the Ar XII 1018/S X $(1196+1213)$ line ratio as a function of height. Since Ar and S are both high-FIP elements, their relative abundance should be photospheric. The electron temperature remains more or less constant with height at $6.24 \leq \log \mathrm{T} \leq 6.26$. Electron temperature and density are shown in Figure 4 (top). 

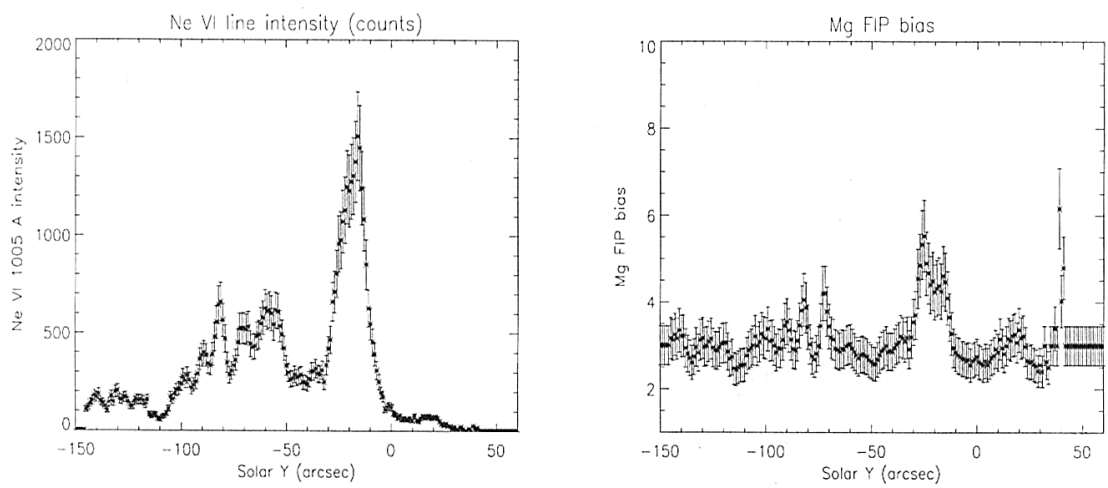

Figure 3. "Prominence" plasma diagnostics. Left: Ne VI line intensity, showing the presence of plasma structures; Right: $\mathrm{Mg} / \mathrm{Ne}$ FIP bias. The $\mathrm{X}$-axis represents the position along the slit. These measurements have been taken at solar $\mathrm{X}=45$.
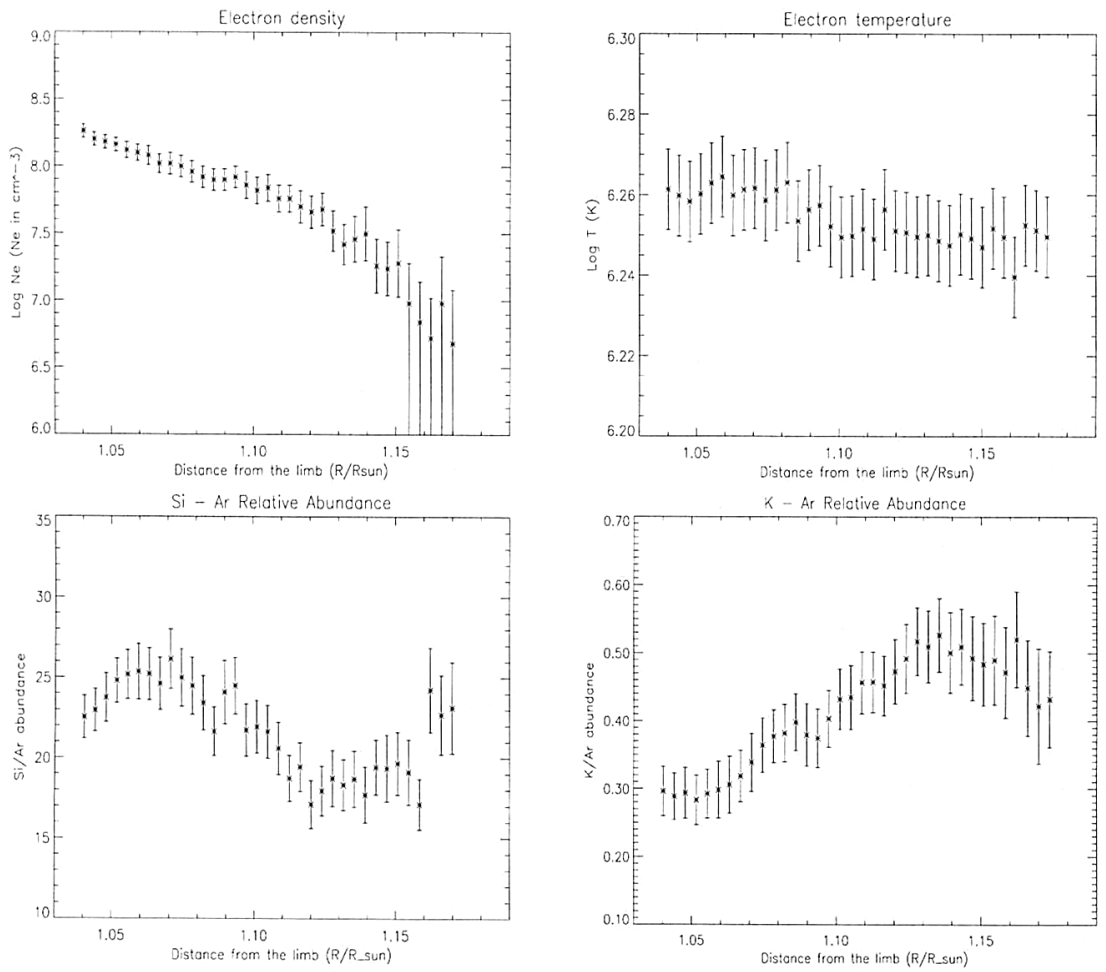

Figure 4. Active corona diagnostics. Top left: $\log \mathrm{N}_{e}$ versus height; Top right: $\log \mathrm{T}$ versus height; Bottom left: $\mathrm{Si} / \mathrm{Ar}$ abundance versus height. Bottom right: $\mathrm{K} / \mathrm{Ar}$ abundance versus height. 
The low-FIP/high-FIP pairs S/Ar, Si/Ar and K/Ar were studied as a function of height. The FIPs for K, Si, S and Ar are 4.3, 8.2, 10.4 and $15.8 \mathrm{eV}$ respectively. The $\mathrm{K} / \mathrm{Ar}, \mathrm{Si} / \mathrm{Ar}$ and $\mathrm{S} / \mathrm{Ar}$ abundances outside the structures show different behaviours. The coronal relative abundance of S/Ar stays almost at its photospheric value; Si/Ar shows a FIP bias of 2 to $3 ; \mathrm{K} / \mathrm{Ar}$ shows a FIP bias increasing with height from 6.4 to 14 . The K XIII line is blended with a Si VIII $n=3 \rightarrow n-3$ transition, whose emission has been taken care of by using Bhatia and Landi (2003) Si VIII calculations. The Si/Ar and K/Ar FIP biases as a function of height are shown in Figure 4 (bottom).

The sulphur FIP bias is almost negligible, showing that $\mathrm{S}$ abundance is photospheric within uncertainties, and does not change with height. Si instead shows a typical FIP bias magnitude, that seems to become smaller by $30 \%$ as height increases, although the most distant (and uncertain) three values contradict this. The K FIP bias is very high, higher than the values quoted in the literature for the other low-FIP elements. The FIP bias for the very low-FIP elements such as $\mathrm{K}$ could be higher than for the normal low-FIP elements such as $\mathrm{Mg}$, Si and Fe, so that the FIP effect for low-FIP elements could be dependent on the FIP itself. The evident height dependence of the K FIP bias is a new observational fact whose magnitude is larger than the combined experimental and theoretical uncertainties.

\section{Conclusions}

We find that the $\mathrm{Mg} / \mathrm{Ne}$ relative abundance is highly variable in the complex, cool core of the active region, strongly correlated with line intensity and magnetic structures. $\mathrm{Mg}$ abundance enhancements relative to Ne reach up to a factor of 8.8. The analysis of the diffuse corona seems to suggest that in off-limb active region plasma the FIP bias inside the low-FIP elements' class is dependent on the FIP value, being higher for the very-low FIP element K. Also, some dependence of the FIP bias with height is suggested. The FIP bias vs. FIP problem is still open and more work is underway.

Acknowledgments. The IAU/INSA travel grant enabled B.N. Dwivedi to present this work at the IAU Symposium 219. A.Mohan acknowledges the support from the SERC/DST, New Delhi. The work of E.Landi is supported by the NASA Living with a Star program.

\section{References}

Bhatia, A.K., Landi, E. 2003, ADNDT, in press

Dere, K.P., Landi, E., Mason H.E., Monsignori Fossi B.C., \& Young P.R. 1997, A\&AS 125,149

Feldman, U., \& Widing, K.G. 2003, Space Sci. Rev., 107, 665

Wilhelm K., et al. 1995, Solar Phys. 162, 189 